\title{
CHARACTERIZATION OF THE INNER DISK AROUND HD 141569 A FROM KECK/NIRC2 L-BAND VORTEX CORONAGRAPHY
}

\author{
Dimitri Mawet ${ }^{1,2}$, Élodie Choquet ${ }^{2,13}$, Olivier Absil ${ }^{3,14}$, Elsa Huby ${ }^{3,15}$, Michael Bottom ${ }^{1,2}$, Eugene Serabyn ${ }^{2}$, \\ Bruno Femenia ${ }^{4}$, Jérémy Lebreton ${ }^{5}$, Keith Matthews ${ }^{1}$, Carlos A. Gomez Gonzalez ${ }^{3}$, Olivier Wertz ${ }^{3,6}$, \\ Brunella Carlomagno $^{3}$, Valentin Christiaens ${ }^{3,7}$, Denis Defrère ${ }^{3,8}$, Christian Delacroix ${ }^{3,9}$, Pontus Forsberg ${ }^{10}$, \\ Serge Habraken ${ }^{3}$, Aissa Jolivet ${ }^{3}$, Mikael Karlsson ${ }^{10}$, Julien Milli ${ }^{11}$, Christophe Pinte ${ }^{12}$, Pierre Piron ${ }^{3,10}$, \\ Maddalena Reggiani ${ }^{3}$, Jean Surdej ${ }^{3}$, and ERnesto Vargas Catalan ${ }^{10}$ \\ ${ }^{1}$ Department of Astronomy, California Institute of Technology, 1200 East California Boulevard, MC 249-17, Pasadena, CA 91125 USA; dmawet@astro.caltech.edu \\ 2 Jet Propulsion Laboratory, California Institute of Technology, 4800 Oak Grove Drive, Pasadena, CA 91109, USA \\ ${ }^{3}$ Space sciences, Technologies and Astrophysics Research (STAR) Institute, Université de Liège, 19 Allée du Six Août, B-4000 Liège, Belgium \\ ${ }^{4}$ W. M. Keck Observatory, 65-1120 Mamalahoa Hwy., Kamuela, HI 96743, USA \\ ${ }^{5}$ NASA Exoplanet Science Institute, California Institute of Technology, 770 South Wilson Avenue, Pasadena, CA 91125, USA \\ ${ }_{7}^{6}$ Argelander-Institut für Astronomie, Auf dem Hügel 71, D-53121 Bonn, Germany \\ ${ }^{7}$ Departamento de Astronomía, Universidad de Chile, Casilla 36-D, Santiago, Chile \\ ${ }^{8}$ Steward Observatory, Department of Astronomy, University of Arizona, 933 North Cherry Avenue, Tucson, AZ 85721, USA \\ ${ }^{9}$ Sibley School of Mechanical and Aerospace Engineering, Cornell University, Ithaca, NY 14853, USA \\ ${ }^{10}$ Department of Engineering Sciences, Ångström Laboratory, Uppsala University, Box 534, SE-751 21 Uppsala, Sweden \\ ${ }^{11}$ European Southern Observatory, Alonso de Cordóva 3107, Vitacura, Santiago, Chile
Univ. Grenoble Alpes, IPAG, F-38000 Grenoble, France CNRS, IPAG, F-38000 Grenoble, France \\ Received 2016 September 22; revised 2016 October 31; accepted 2016 November 16; published 2017 January 4
}

\begin{abstract}
HD $141569 \mathrm{~A}$ is a pre-main sequence B9.5 Ve star surrounded by a prominent and complex circumstellar disk, likely still in a transition stage from protoplanetary to debris disk phase. Here, we present a new image of the third inner disk component of HD 141569 A made in the $L^{\prime}$ band $(3.8 \mu \mathrm{m})$ during the commissioning of the vector vortex coronagraph that has recently been installed in the near-infrared imager and spectrograph NIRC 2 behind the W.M. Keck Observatory Keck II adaptive optics system. We used reference point-spread function subtraction, which reveals the innermost disk component from the inner working distance of $\simeq 23$ au and up to $\simeq 70$ au. The spatial scale of our detection roughly corresponds to the optical and near-infrared scattered light, thermal $Q, N$, and $8.6 \mu \mathrm{m}$ PAH emission reported earlier. We also see an outward progression in dust location from the $L^{\prime}$ band to the $H$ band (Very Large Telescope/SPHERE image) to the visible (Hubble Space Telescope (HST)/STIS image), which is likely indicative of dust blowout. The warm disk component is nested deep inside the two outer belts imaged by HST-NICMOS in 1999 (at 406 and 245 au, respectively). We fit our new $L^{\prime}$-band image and spectral energy distribution of HD 141569 A with the radiative transfer code MCFOST. Our best-fit models favor pure olivine grains and are consistent with the composition of the outer belts. While our image shows a putative very faint point-like clump or source embedded in the inner disk, we did not detect any true companion within the gap between the inner disk and the first outer ring, at a sensitivity of a few Jupiter masses.
\end{abstract}

Key words: planet-disk interactions - planetary systems - planets and satellites: formation - protoplanetary disks stars: pre-main sequence - stars: variables: T Tauri, Herbig $\mathrm{Ae} / \mathrm{Be}$

\section{INTRODUCTION}

The study of the morphology and composition of circumstellar disks in systems of different ages and masses allows us to probe different stages of the formation and evolution of planetary systems in diverse environmental conditions. This endeavor is complementary to the search of exoplanets, characterization of outstanding extra-solar planetary systems, and planet population demographics. These synergistic approaches should allow us to relate the diversity of planetary systems observed by large-scale surveys to the initial conditions of stellar and planet formation, the ultimate goal of comparative exoplanetology.

HD 141569 A, a B9.5 Ve pre-main sequence star in a triple system $116 \pm 8 \mathrm{pc}$ from the Sun (van Leeuwen 2007), is a natural testbed for stellar and planetary system evolution

\footnotetext{
13 Hubble fellow.

14 F.R.S.-FNRS Research Associate.

15 F.R.S.-FNRS Postdoctoral Researcher.
}

theories (Table 1). It is a young star with an age estimate of $5 \pm 3 \mathrm{Myr}$ (Weinberger et al. 1999), and is understood to be undergoing its transition from protoplanetary disk to debris disk. Using (Hubble Space Telescope) HST-NICMOS imaging, Augereau et al. (1999) and Weinberger et al. (1999) almost simultaneously reported the discovery of two massive rings of dust around HD $141569 \mathrm{~A}$. The two resolved outer rings are roughly delineated in Figure 1 by the green ellipses. Clampin et al. (2003) reported a fractional infrared excess luminosity of $L_{\text {disk }} / L_{\text {star }}=8.4 \times 10^{-3}$, three times that of the $\beta$ Pictoris debris disk. Thi et al. (2014) reported a disk-averaged gas-todust ratio of $\simeq 100$, close to the initial interstellar value. All available empirical evidence clearly indicates that HD $141569 \mathrm{~A}$ is still transitioning from the protoplanetary gas-rich to the canonical gas-poor debris disk phase.

The recent study of HD 141569 A with Gemini/NICI (Biller et al. 2015) reported outer and inner ring radii of $406 \pm 13.3$ au and $245 \pm 3.0$, common position angle of $-11^{\circ} .3$ (resp. $-8^{\circ} .9$ ), and inclination of $43^{\circ} .7$ (resp. 44.9). Biller et al. (2015) find an offset of 4 au between the inner ring center and the stellar 
Table 1

Properties of HD 141569 A

\begin{tabular}{ll}
\hline \hline Properties & Value \\
\hline Coord. (hms, dms) & $154957.74785-035516.3430$ \\
Spectral type & B9.5 Ve \\
Distance (pc) & $116 \pm 8 \mathrm{pc}$ \\
$V$ mag & 7.2 \\
$L^{\prime}$ mag & 6.83 \\
$L^{\prime}$ flux (Jy) & 0.46 \\
$T_{\text {eff }}(\mathrm{K})$ & 10,000 \\
Age & $5 \pm 3 \mathrm{Myr}$ \\
\hline
\end{tabular}

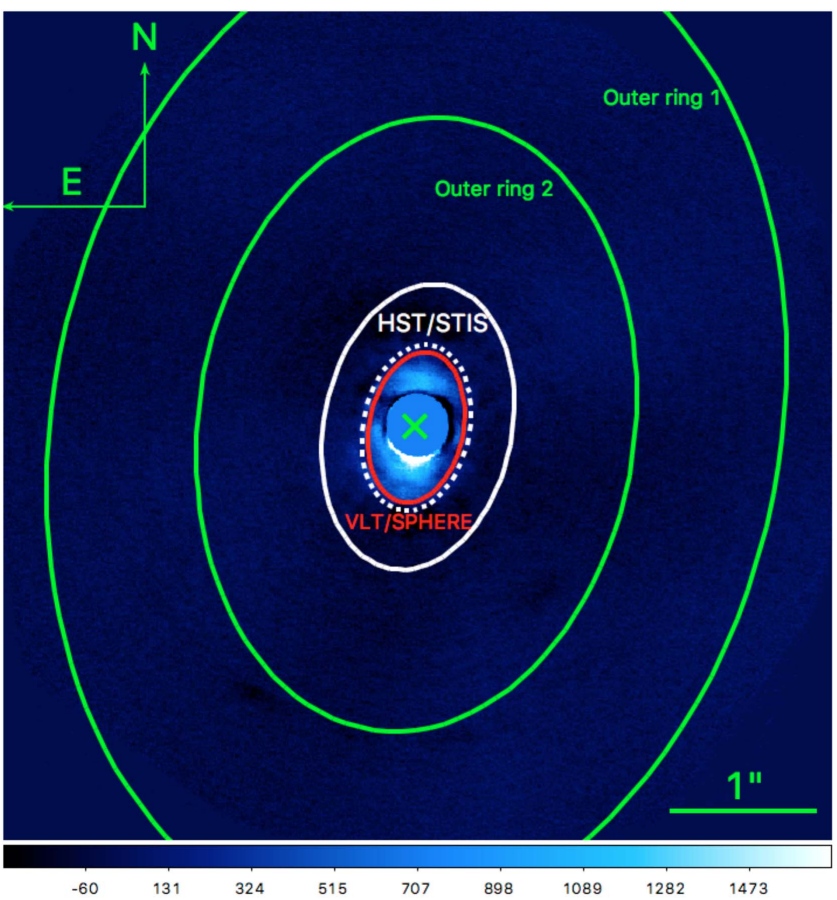

Figure 1. Wide field view of HD 141569 A from our $L^{\prime}$-band vortex coronagraph data, showing the trace of the outer rings, not detected here. The inner white plain and dashed ellipses approximately delineate the disk component detected by HST/STIS (Konishi et al. 2016), clearly outside our detection. The red ellipse shows the approximate location of the main brightest inner ring reported by Perrot et al. (2016) using VLT/SPHERE. The color scale in the image is linear and analog to digital units (ADU).

position, possibly hinting at the presence of unseen companion. This result has been disputed by a reanalysis of the same NICI data set combined with HST/NICMOS data by Mazoyer et al. (2016), however, who found no significant offset. Biller et al. (2015) also report an additional arc-like feature between the inner and outer ring that is only evident on the east side, and claims an evacuated cavity from 175 au inwards.

The discovery of the third innermost warm disk component of HD 141569 A was first reported in Fisher et al. (2000) at 10.8 and $18.2 \mu \mathrm{m}$, using OSCIR on Keck. The mid-infrared emission from the warm disk was also confirmed later on by Marsh et al. (2002), and was detected out to a radius of $100 \mathrm{au}$, well inside the two outer belts (Figure 1), with a profile and brightness reminiscent of the debris disks around HR 4796A and $\beta$ Pictoris. Fisher et al. (2000) used the 10.8 and $18.2 \mu \mathrm{m}$ images to place a lower limit of $170 \mathrm{~K}$ on the temperature and an upper limit of $2 \mu \mathrm{m}$ on the diameter of the dust grains responsible for the mid-IR emission, assuming astronomical silicates. Fisher et al. (2000) argue that contamination of the
Table 2

Observing Log

\begin{tabular}{ll}
\hline \hline Properties & Value \\
\hline UT date (yyyy mm dd)) & 2015 Nov 06 \\
UT start time (hh:mm:ss) & $08: 10: 55$ \\
UT end time (hh:mm:ss) & $10: 04: 44$ \\
Discr. Int. Time (s) & 0.2 \\
Coadds & 100 \\
Number of frames & 39 \\
Total integration time (s) & 780 \\
Plate scale (mas/pix) & 9.942 ("narrow") \\
Total FoV & $r \simeq 5^{\prime \prime}$ (vortex mount) \\
Filter & $L^{\prime}$ \\
Coronagraph & Vortex (AGPM) \\
Lyot stop & Inscribed circle \\
Reference PSF & HD 144271 \\
Median seeing $\left({ }^{\prime \prime}\right)$ & 0.5 \\
Par. angle start-end $\left({ }^{\circ}\right)$ & -24 to +26 \\
Mean airmass & 1.1 \\
\hline
\end{tabular}

mid-infrared flux by polycyclic aromatic hydrocarbon (PAH) emission marginally affects the derived grain size. Thi et al. (2014) discuss 2005 VISIR data obtained in the PAH filter at $8.6 \mu \mathrm{m}$, and report the detection of infrared emission overlapping with the warm disk of Fisher et al. (2000) and Marsh et al. (2002). The VISIR image of the warm inner disk component was interpreted as emission from PAHs by Thi et al. (2014). The fitted disk-averaged gas-to-dust-mass ratio is about 100 , with a gas mass of $4.9 \times 10^{-4} M_{\odot}$ and accounts for multiwavelength data, in particular Herschel PACS spectra. This result implies that if the disk was originally massive, the gas and the dust would have dissipated at the same rate.

Very recently, Konishi et al. (2016) presented a HST/STIS image of a new disk component at 65-100 au around HD $141569 \mathrm{~A}$, nested inside the two outer belts (see the dashed and plain white contours in Figure 1). This optical image seems at first sight to be the scattered-light counterpart of the known resolved thermal emission discussed above. We show below that there is very little overlap, if any, between the thermal emission and the scattered-light HST/STIS component reported in Konishi et al. (2016). The latter would thus be tracing a fourth disk component and dust population.

We also note two recent independent detections of complex inner disk features from Currie et al. (2016) and Perrot et al. (2016). Currie et al. (2016) reported the detection in the $L^{\prime}$ band, using Keck/NIRC2 in saturated mode and using angular differential imaging (ADI). Perrot et al. (2016) presented $Y-, J-$, $H$-, and $K s$-band images obtained with the second-generation extreme-adaptive optics planet-finder SPHERE at the Very Large Telescope (VLT), also using ADI. These detections are, by nature, and as a result of the disk geometry and complex morphology, affected by observational biases (Milli et al. 2012). Here we present a new coronagraphic $L^{\prime}$-band image with high signal-to-noise ratio obtained with the near-infrared imager and spectrograph NIRC2 at W.M. Keck observatory.

\section{OBSERVATIONS AND DATA REDUCTION}

We observed HD 141569 A on 2015 June 11 (see Table 2), during the commissioning of the new $L^{\prime}$-band vector vortex coronagraph installed in NIRC2 (Serabyn et al. 2016), the nearinfrared camera and spectrograph behind the adaptive optics system of the Keck II telescope at W.M. Keck Observatory. 
The vortex coronagraph is a phase-mask coronagraph enabling high-contrast imaging at very small angles close to the diffraction limit of the $10 \mathrm{~m}$ Keck telescope at $3.8 \mu \mathrm{m}$ $(\simeq 0$ ". 08$)$. The starlight suppression capability of the vortex coronagraph is induced by a $4 \pi$ radian phase ramp wrapping around the optical axis. When the coherent adaptively corrected point-spread function (PSF) is centered on the vortex phase singularity, the on-axis starlight is redirected outside the image of the telescope pupil formed downstream from the coronagraph, where it is blocked by means of an undersized diaphragm (the Lyot stop). The vector vortex coronagraph installed in NIRC2 was made from a circularly concentric subwavelength grating etched onto a synthetic diamond substrate (Annular Groove Phase Mask coronagraph or AGPM, Mawet et al. 2005; Delacroix et al. 2013). The mask is nearly identical to its siblings installed inside NACO at the VLT (Absil et al. 2013; Mawet et al. 2013) and LMIRCam at the Large Binocular Telescope (Defrère et al. 2014). See also Vargas Catalan et al. (2016) for more details about the AGPM technology and manufacturing process.

Observing conditions were very good, with optical median seeing $\simeq 0$." 5 (estimated from the adaptive optics system telemetry). The adaptive optics system provided excellent correction in the $L^{\prime}$ band with a Strehl ratio in excess of $85 \%$, similar to the image quality provided at shorter wavelengths by more recent extreme adaptive optics systems such as the Gemini Planet Imager and VLT/SPHERE.

The alignment of the star onto the coronagraph center, a key to high contrast at small angles, was performed using a new method named quadrant analysis of coronagraphic images for tip-tilt sensing (QACITS, Huby et al. 2015). The QACITS pointing control uses NIRC2 focal-plane coronagraphic science images in a closed feedback loop with the Keck adaptive optics tip-tilt mirror (through the real-time update of Shack-Hartman centroid offsets) to guarantee optimal centering at all times (Serabyn et al. 2016). It also significantly improves reproducibility and stability of the centering onto the vortex between targets (E. Huby et al. 2016, in preparation). The typical centering accuracy provided by QACITS is $\simeq 0.025 \lambda / D \mathrm{rms}$, or $\simeq 2$ mas rms. We observed a calibrator star, HD 144271, which is an A0 star with similar $V$ and $L$ magnitude to HD 141569 A. We switched back and forth between target and reference star every 20 minutes. Reacquisition overheads when switching from the target to the reference and vice versa were minimized to less than two minutes (including recentering behind the coronagraph).

The data were reduced by dividing the images by a sky flat field obtained without the vortex phase mask. No sky background image was subtracted as this unnecessarily introduces additional noise. Instead, we subtracted a reference PSF constructed by projecting the target images on a subset of the Karhunen-Loève basis (five first principal components) of the reference star. Reference star differential imaging (RDI) with principal component analysis (PCA-RDI) is very effective in the high Strehl regime provided that the duty cycle between the target and reference star beats speckle decorrelation timescales for a given target contrast (Mawet et al. 2009, 2011; Serabyn et al. 2010; Soummer et al. 2012). It also is immune to the self-subtraction phenomenon that affects angular and spectral differential imaging (ADI/SDI), especially at small inner working angles. It might be subject to oversubtraction, however, which could impact photometric precision (Pueyo 2016).

To verify and ensure that oversubtraction is indeed minimum, we also used a classical reference star PSF subtraction (c-RDI) by computing the median image of the reference target data cube (we excluded the $10 \%$ of the reference frames that are the least correlated to the science frames), and removing it from the target star frames, with a scaling coefficient equal to its projection on the target star frames. This process reveals the inner disk with a similar image quality as when using PCA. Our final reduced images with PCA-RDI and c-RDI are shown in Figure 2, left and right, respectively. The two reduced images are virtually the same, which indicates that the Keck $L^{\prime}$-band PSF is very stable. The differences between ADI and RDI can be seen by comparing this image to the ADI-reduced image of Currie et al. (2016). Our RDI image is not affected by typical ADI self-subtraction biases (similar to high-pass filtering), and so the fainter and more extended disk components are revealed, extending out to $\simeq 0$ " 6 . In particular, our RDI image appears more ring-like, as opposed to two point-like peaks at each side of the star.

\section{DETECTION OF THE THIRD INNER DISK COMPONENT}

We detect the third disk component to the complex debris disk structure around HD 141569 A. This disk component is the innermost of the three so far discovered components in the HD 141569 A system (Figures 1 and 2), and is detected from our effective inner working angle of 0 .' 2 up to 0 !. 6 , corresponding to 23 and $70 \mathrm{au}$, respectively. This range also roughly coincides with the resolved thermal emission, so that the $L^{\prime}$-band image seems to show the short-wavelength counterpart of the extended thermal feature detected in the mid-infrared ( $Q$ and $N$ bands) by Keck/OSCIR, Keck/ MIRLIN, and VISIR more than a decade ago (Fisher et al. 2000; Marsh et al. 2002; Thi et al. 2014).

The vortex coronagraph has a theoretical inner working angle of $0.9 \lambda / D \simeq 0$. 07 , with the wavelength $\lambda=3.8 \mu \mathrm{m}$ and the telescope diameter $D \simeq 10 \mathrm{~m}$. Owing to the central obscuration, segmented pupil geometry, and Lyot stop size, the Keck/NIRC2 vortex has a measured inner working angle of $1.6 \lambda / D \simeq 0$ ". 125 . The-effective-inner working angle is affected by the residuals caused by imperfect reference subtraction, and is for this particular data set closer to $\simeq 2 \lambda / D$.

For typical dust grain composition and size in debris disks, the equilibrium temperature at the separations probed by our observations is $\simeq 100 \mathrm{~K}$, which is warmer than typical cold Kuiper belts found by Spitzer or Herschel at $\leqslant 50 \mathrm{~K}$, but not as warm as asteroid belt dust $(\sim 150 \mathrm{~K})$ or zodiacal dust $(\sim 300 \mathrm{~K})$ in the solar system. The $3.8 \mu \mathrm{m}$ thermal emission of dust disk models peaks at a radius much smaller than observed, pointing to a scattered-light origin for the disk component detected at $L^{\prime}$. Moreover, the brightness profiles extracted over the disk major axis (using aperture photometry with a radius $r=\lambda / D \approx 0$ !" 08 ) presented in Figure 3 show a steep $r^{-4}-r^{-5}$ power law between 23 and $70 \mathrm{au}$, compared to the $\simeq r^{-2.5}$ of the VISIR $8.6 \mu \mathrm{m}$ PAH image of Thi et al. (2014). The slope difference implies that the disk should become redder at larger separations. However, PAH emission is an unreliable tracer of dust, and we chose not to make direct comparisons between these images. 


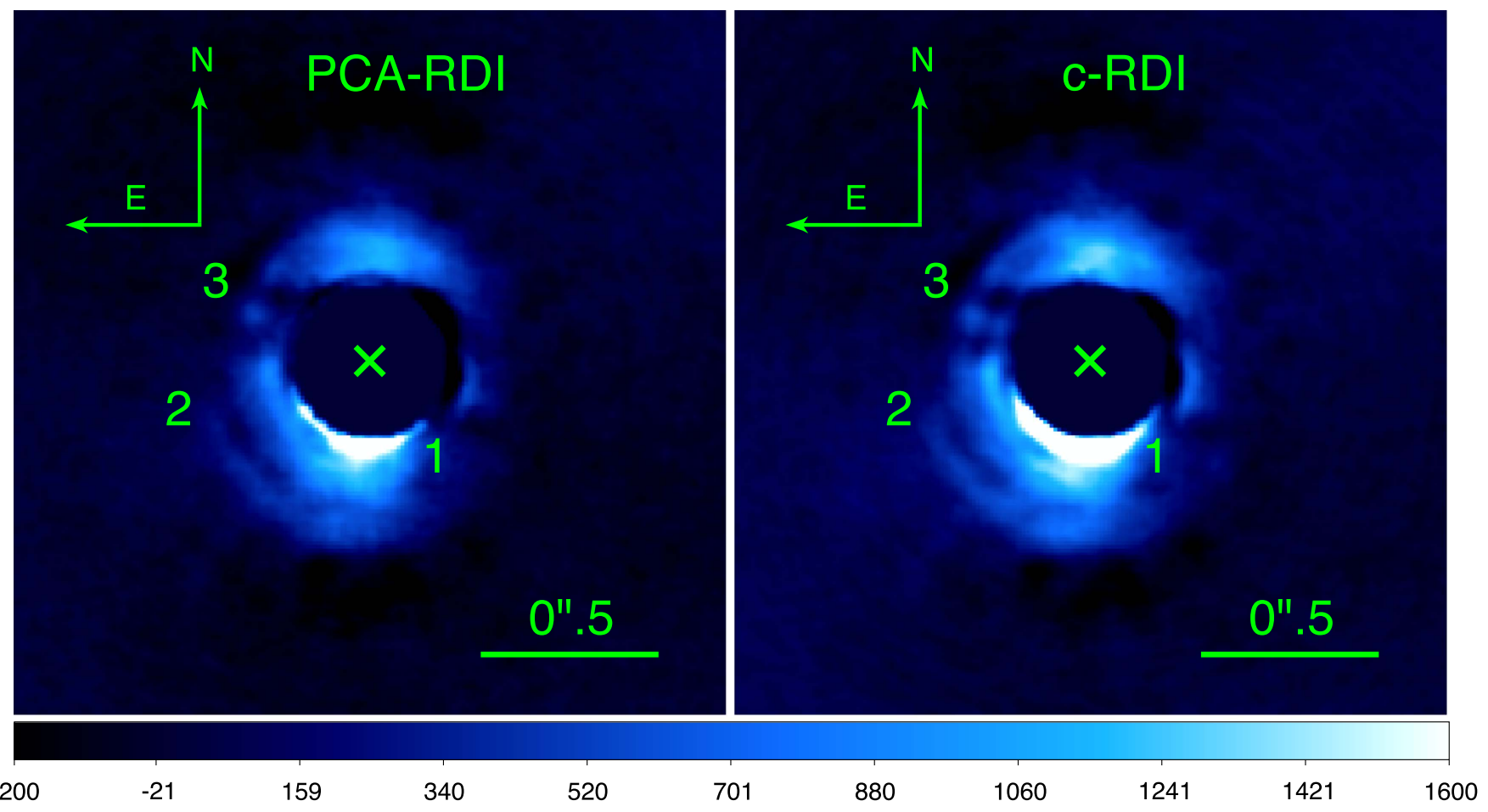

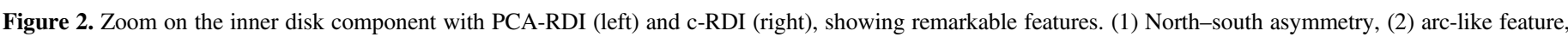
(3) point-like source seemingly embedded in a gap in the ring. The color scale in both images is linear and analog to digital units (ADU).

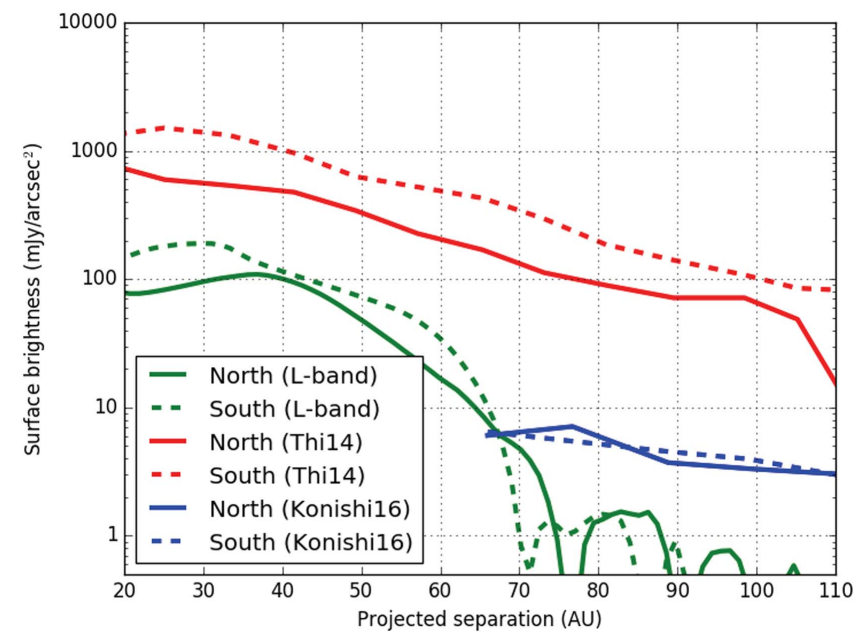

Figure 3. Surface brightness profiles $\left(\mathrm{mJy} / \operatorname{arcsec}^{2}\right)$ of the disk extracted along its major axis, and using aperture photometry with a radius $r=\lambda / D \approx 0$." 08 , toward the north (plain curve) and south (dashed curve), respectively. The green curve shows our $L^{\prime}$-band data. We overplot the published VISIR $8.6 \mu \mathrm{m}$ PAH profile from Thi et al. (2014) in red and the published HST/STIS optical profile from Konishi et al. (2016) in blue.

We note that both the PCA-RDI and c-RDI images show three remarkable features (noted 1, 2, and 3 in Figure 2), although we recognize that they could be artifacts resulting from imperfect coronagraph centering. Indeed, the data were taken during the vortex coronagraph commissioning run and the pointing was thus at times suboptimal (e.g., the QACITS set point was likely not fully consistent between the target and reference, inducing differential structures mostly within $0 . / 2$, but also potentially slight changes in the diffraction pattern beyond).
The first feature is a noticeable N-S asymmetry (the south side being about twice as bright as the north side, see Figure 3) that could also be due to pointing errors on the coronagraph. However, the feature is also seen in Currie et al. (2016) and follows the N-S asymmetry of the ring "R1" identified in the SPHERE images at shorter wavelengths by Perrot et al. (2016), which reveals a fine ring traced to the south, almost invisible to the north.

The second interesting feature is an arc-like structure to the southeast at a radius $r \simeq 0$ ". 6 . A similar feature is reported in Currie et al. (2016), and in the near-infrared SPHERE images. In the SPHERE images, it is identified as ring "R2" (Perrot et al. 2016). It is also seen in the optical HST/STIS image, and is identified as an "arc-like" feature (Konishi et al. 2016).

The third feature is a point-like structure to the east $(\mathrm{FWHM} \approx 1 \lambda / D)$. The projected angular separation is 0 "' $330 \pm 0.010$, corresponding to a projected physical separation of $\simeq 38$ au and position angle of $70^{\circ} \pm 2$. Using aperture photometry with an aperture radius $r=\lambda / D$, we find that this point-like feature is marginally detected at a signal-to-noise ratio of $\simeq 2$, and $\simeq 9$ mag fainter than the central star in the $L^{\prime}$ band $\left(L^{\prime}=15.8_{-0.45}^{+0.75}\right)$. The low-significance detection could also be a dust clump embedded in the inner disk component. We also note that while other point-like feautres are seen in their images, our particular detection is not reported in the saturated Keck/NIRC2 data set of Currie et al. (2016), nor in the SPHERE near-infrared images of Perrot et al. (2016).

\section{DISK MODELING}

In addition to characterizing the morphology of the inner disk around HD $141569 \mathrm{~A}$, this new image in the $L^{\prime}$ band allows us to place some constraints on the dust composition of the system, as we discuss in this section. Although the model proposed by Thi et al. (2014) provides a good fit to the $8.6 \mu \mathrm{m}$ 
PAH emission image and to the spectral energy distribution (SED), it fails at properly reproducing our $L^{\prime}$-band image of the inner disk component, which is expected. Without an image of the dust continuum, there is indeed a strong degeneracy between the disk morphology and composition parameters, especially for a complex multibelt system such as HD 141569 A.

To characterize the morphology and composition of the inner disk component, we proceeded in two steps with complementary modeling tools. First, we constrained the geometry of the inner disk using the GRaTer ray-tracing code (Augereau et al. 1999; Lebreton et al. 2012), which creates scattered-light images of optically thin debris disk models assuming simple anisotropic scattering (Henyey \& Greenstein 1941). These simplified models allow us to estimate offsets of the disk with respect to the star and compare them with previously published measurements. We then used the Monte Carlo radiative transfer code MCFOST (Pinte et al. 2006, 2009) to analyze the morphology and composition of the complete dust system altogether by fitting models to both our $L^{\prime}$-band image and the system SED.

\subsection{Modeled Data Sets}

We fit our models to the $L^{\prime}$-band image of the disk obtained after a classical reference star PSF subtraction (c-RDI, see Section 2). When computing goodness-of-fit estimators, we masked out the pixels outside of an ellipse centered on the disk of semiminor and semimajor axes 0 ." 57 and 0 ." 66 , respectively, to consider only the area where the disk is detected as well as a thin $\sim 1 \lambda / D$-wide ring around the disk where it is not detected. We also masked out the inner part of the image within a radius of 0 "'22, leaving a total of $N_{\mathrm{im}}=10286$ pixels in the fit process.

To estimate the noise map used in the least-squares fits, we adapted the procedure described in Choquet et al. (2016), and proceeded as follows: (1) we estimated the noise on a single NIRC2 exposure by reducing the 23 images of the reference star with the same method as used for HD $141569 \mathrm{~A}$, then computing the "temporal" standard deviation for each pixel through these reference images; (2) we then estimated the noise in the final image of HD 141569 A (which combines 36 exposures rotated to north up) by adding in quadrature 36 replicas of the single-exposure noise map, derotated by the same parallactic angles as for the HD 141569 A frames. This method allows us to estimate the local noise in the image (including smearing of speckle noise caused by the derotation and combination of images over a large parallactic angle range), even in regions with extended astrophysics signal, as opposed to classical radial noise curves estimated from spatial noise in the science image itself.

As a sanity check, we also estimated the background noise based on the photon counts in our image, and we found a similar noise level as in our noise map. This demonstrates that our reduced image is mostly dominated by background noise, except for close to the star, where the noise source is a combination of speckle noise and background noise, which is accurately captured by our temporal noise calculation.

In Section 4.3 we additionally use the system SED to further constrain the dust characteristics in HD $141569 \mathrm{~A}$. We used the photometric data at wavelengths longer than $5 \mu \mathrm{m}$ listed in Thi et al. (2014), binned to have as many photometric points per decade $\left(N_{\mathrm{SED}}=19\right.$ data points). To account for underestimated error bars, cross-calibration systematics between instruments, and natural variability, we set all the uncertainties to $10 \%$ in the fitting process. The SED photometric measurements are presented in Figure 4. The stellar photometry was corrected for reddening based on Cardelli et al. (1989) and using O'Donnell (1994) updated constants for the near-UV.

\subsection{Geometry of the Inner Disk}

To constrain the most relevant morphological parameters of the inner disk component, we created a grid of $~ 1300$ models with the GRaTer code, using the inclination, position angle, parent dust belt radius, disk offsets, and degree of forward scattering as free parameters. We used a Gaussian profile for the vertical dust density distribution, with a linear vertical scale height of an opening angle of 5\% (Thi et al. 2014). We simulated the surface density distribution of the disk with a combination of two radial power laws, with slopes arbitrarily set to $\alpha_{\text {in }}=3$ and $\alpha_{\text {out }}=-5$, respectively, inward and outward from the parent belt radius. As Currie et al. (2016), we found that these parameters are not constrained by our data. The total flux of the normalized models are adjusted to the data using a linear regression with the observed disk.

The parameters of the best model are reported in Table 3 and correspond to an almost isotropic disk (Henyey-Greenstein asymmetric scattering factor $g=0.1$ ), with a parent belt radius of 39 au $(\sim 0$ "! 34$)$ inclined by $53^{\circ}$ with a position angle of $-11^{\circ}$ north to east. These values are consistent with the corresponding parameters for the two outer rings, both regarding the geometrical parameters (Biller et al. 2015) and the anisotropy of the dust grains (Weinberger et al. 1999). We note that our best-fit geometry differs from the characteristics estimated by Currie et al. (2016), although we used the same modeling code. For instance, we estimate the disk offsets from the star to be $x_{c}=-2 \pm 7$ au and $y_{c}=0 \pm 4$ au in the detector frame, which are consistent with a belt centered on the star position. Our $x_{c}=-2 \pm 7$ au value significantly differs from the 7.9 au offset found by Currie et al. (2016).

One possible explanation for the discrepancy is that Currie et al. (2016) used a fixed -1.2 position angle value, and their best-fit $56^{\circ}$ inclination hits the lower limit of their model grid. However, it is worth noting that Mazoyer et al. (2016) did not find any significant offset for the two outermost rings in their reanalysis of Gemini/NICI and HST/NICMOS data, in disagreement with Biller et al. (2015).

\subsection{Detailed Morphology and Chemical Composition of the Inner Disk}

To place constraints on the dust composition in the HD 141569 A disk system, we fit our $L^{\prime}$-band image and the system SED to models generated with the radiative transfer code MCFOST. We computed a grid of 194400 models with $N_{\text {par }}=10$ varying parameters to describe the inner disk morphology and composition: the inner and outer radius of the disk $R_{\text {in }}$ and $R_{\text {out }}$, the vertical scale height $H_{0}$ at 100 au from the star, the surface density exponent $\alpha$, the flaring exponent $\beta$, the dust mass $M_{\text {dust }}$, the minimum and maximum grain sizes $a_{\min }$ and $a_{\max }$, the grain porosity $p$, and the dust composition. Neglecting pressure drag from the gas, we fixed the dust size distribution to the standard collisional cascade power law with exponent -3.5 from Dohnanyi (1969). We adopted homogeneous spherical dust grains, and used the Mie theory to 

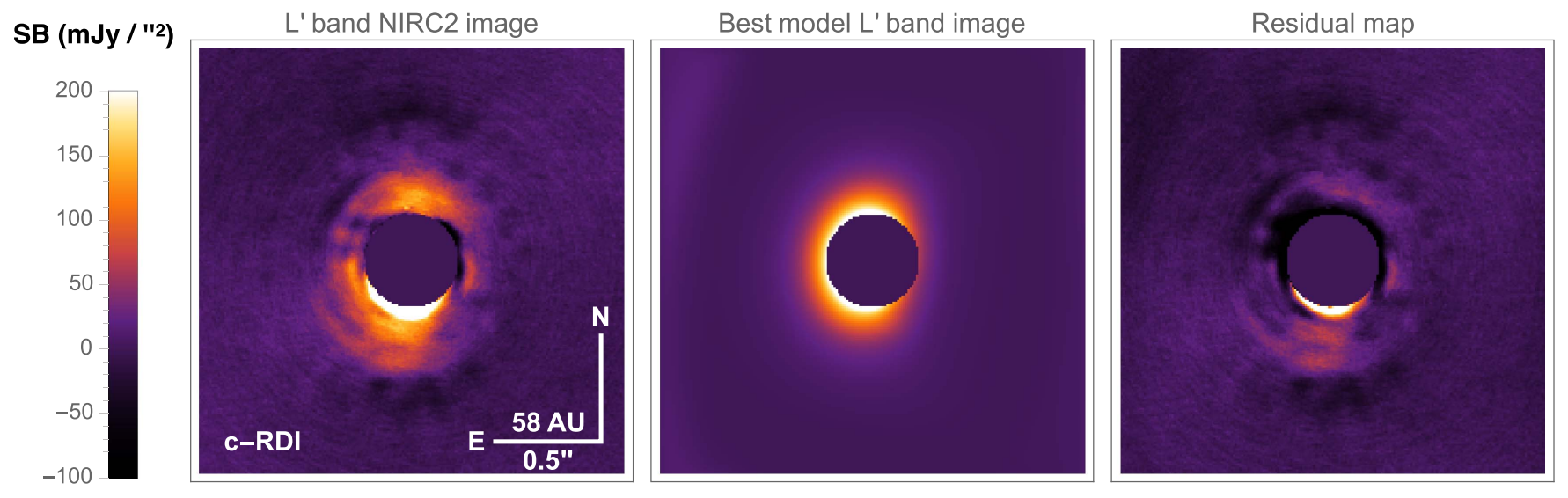

Observations and best model SEDs

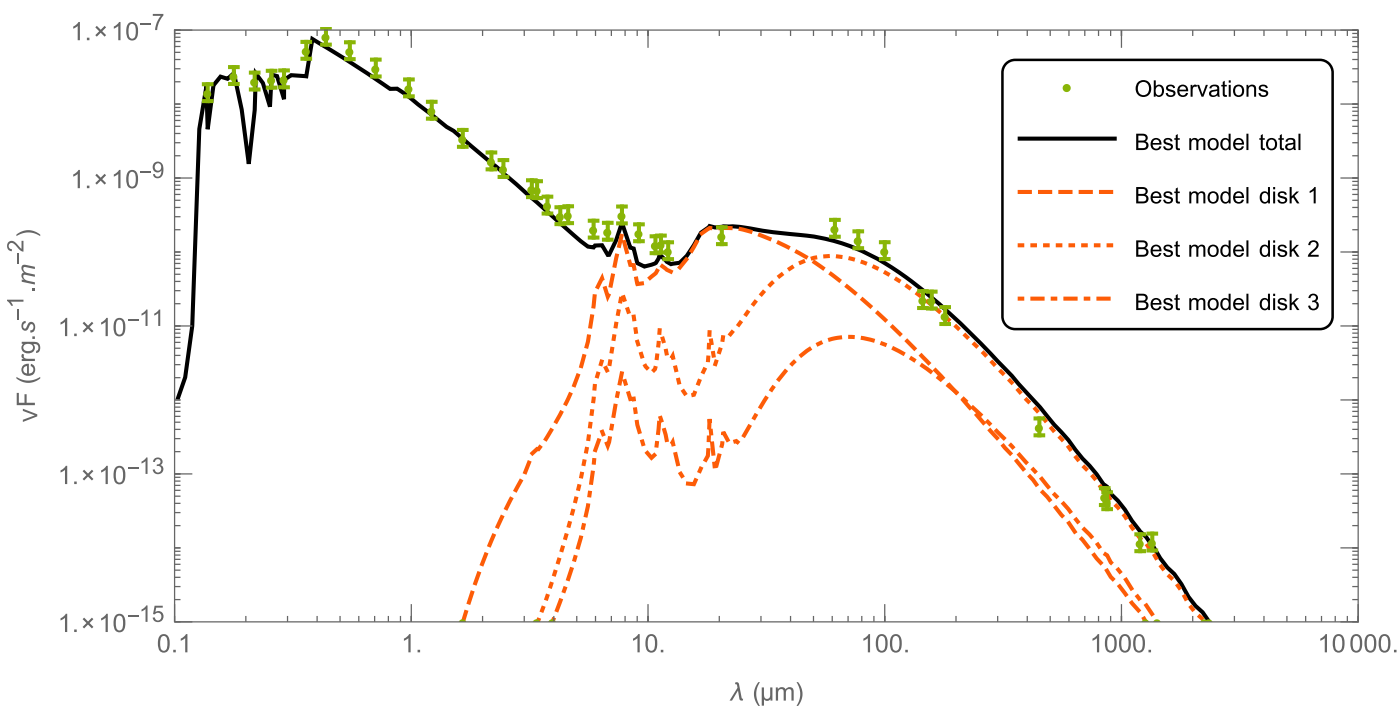

Figure 4. Most likely model fitting both our $L^{\prime}$-band image of HD 141569 A innermost disk and its SED altogether. The top row shows the $L^{\prime}$-band image of HD 141569 A obtained with the Keck-NIRC2 vortex coronagraph after classical reference star PSF subtraction (left), the $3.8 \mu \mathrm{m}$ image of the best model (see Table 4) (middle), and the residual map obtained by subtracting the image of the best model from the $L^{\prime}$-band image. All three images are shown with the same field of view and the same linear scaling. The bottom row shows the measured SED of the system (green points), and the SED of the best model (black line). The red lines shows the individual contributions of the three dust belts in the best model.

Table 3

Geometrical Properties of the Innermost Disk Component Around HD 141569 A

\begin{tabular}{lr}
\hline \hline Properties & Model \\
\hline Position angle of major axis $\left({ }^{\circ}\right)$ & $-11 \pm 8$ \\
Parent belt location $r_{0}(\mathrm{au})$ & $39 \pm 4$ \\
Inclination $\left({ }^{\circ}\right)$ & $53 \pm 6$ \\
Disk offset in $x x_{c}(\mathrm{au})$ & $-2 \pm 7$ \\
Disk offset in $y y_{c}(\mathrm{au})$ & $0 \pm 4$ \\
Coeff. of forward scatt. $g$ & $0.1 \pm 0.1$ \\
\hline
\end{tabular}

derive their optical properties: the scattering and absorption opacities, as well as the scattering phase functions. We used four different dust species in our grid of models: pure olivine grains (Dorschner et al. 1995) as used by Thi et al. (2014) to model the three dust belts in HD $141569 \mathrm{~A}$, and three different mixtures of amorphous silicates (Draine \& Lee 1984), amorphous carbon (Rouleau \& Martin 1991), and waterdominated ice (Li \& Greenberg 1998). The three mixtures all have a different dominant component, with the respective proportions 1:2:3 (water-ice dominant), 2:3:1 (carbon dominant), and 3:1:2 (silicate dominant). The parameter values simulated in the model grid are described in Table 4. The morphology and composition of the two outer belts and the PAH content in the inner disk were kept fixed to the values adopted by the best fit derived in Thi et al. (2014).

MCFOST generates SED data points at prescribed wavelengths, as well as synthetic images. The model images at $3.8 \mu \mathrm{m}$ are convolved with the $L^{\prime}$-band Keck PSF measured off-axis during the coronagraphic acquisition sequence. We note that the absolute flux of disks in scattered light is poorly predicted by radiative transfer algorithms because of the poor observational constraints on the dust scattering phase functions at small scattering angles (Hedman \& Stark 2015). Combined fits of unscaled model images to scattered-light images and SEDs usually lead to aberrant results, as we experienced in this study, and as was also reported by Schneider et al. (2006) and Milli et al. (2015). To address this known problem, we used a free scaling coefficient to adjust the flux of the model images to the flux of the observed disk, which is equivalent to relaxing the constraint on the dust mass in the image (Lebreton et al. 2012). 
Table 4

Parameters Probed in Our Grid of 194400 MCFOST Models, and Best Fits to the SED and $L^{\prime}$-band Data

\begin{tabular}{|c|c|c|c|c|c|c|c|}
\hline Parameters & Min. Value & Max. Value & Sampling & $N_{\text {sample }}$ & Best SED & Best image & $\overline{\text { Best Comb. }}$ \\
\hline$M_{\text {dust }}\left(M_{\odot}\right)$ & $0.2 \mathrm{e}-6$ & $3.2 \mathrm{e}-6$ & $\log$. & 5 & $0.2 \mathrm{e}-6$ & $0.2 \mathrm{e}-6^{\mathrm{a}}$ & $0.2 \mathrm{e}-6$ \\
\hline$a_{\min }(\mu \mathrm{m})$ & 0.1 & 10 & $\log$. & 5 & 0.5 & 10 & 0.1 \\
\hline$a_{\max }(\mu \mathrm{m})$ & 1000 & 10000 & $\log$. & 3 & 10000 & 5000 & 10000 \\
\hline$R_{\text {in }}(\mathrm{au})$ & 20 & 40 & lin. & 3 & 20 & 30 & 20 \\
\hline$R_{\text {out }}(\mathrm{au})$ & 70 & 110 & lin. & 3 & 110 & 70 & 90 \\
\hline$H_{0}(\mathrm{au})$ & 5 & 20 & $\log$. & 3 & 5 & 10 & 20 \\
\hline$\alpha$ & -2.5 & -0.5 & lin. & 3 & -2.5 & -0.5 & -0.5 \\
\hline$\beta$ & 1.00 & 1.25 & $\ldots$ & 2 & $1.25^{\mathrm{a}}$ & $1.25^{\mathrm{a}}$ & $1.25^{\mathrm{a}}$ \\
\hline$p(\%)$ & 0.0 & 0.9 & lin. & 4 & 0.0 & 0.3 & 0.0 \\
\hline Dominant species & \multicolumn{3}{|c|}{ olivine, silicate, carbon, water ice } & 4 & Olivine & Olivine & Olivine \\
\hline$\chi_{\mathrm{SED}}^{2}$ & $\cdots$ & $\cdots$ & $\cdots$ & $\cdots$ & 32 & 61 & 35 \\
\hline$\chi_{\mathrm{im}}^{2}$ & $\cdots$ & $\cdots$ & $\ldots$ & $\ldots$ & 22 & 10 & 14 \\
\hline$\chi_{\mathrm{comb}}^{2}$ & $\cdots$ & $\cdots$ & $\cdots$ & $\ldots$ & 54 & 71 & 49 \\
\hline
\end{tabular}

Note.

${ }^{a}$ Not well constrained by our Bayesian analysis. See Figure 5.

To assess the goodness-of fit of our models to the data, we computed three $\chi^{2}$ estimators for each model: a reduced chi square on the SED $\chi_{\mathrm{SED}}^{2}(9$ degrees of freedom), a reduced chi square on the $L^{\prime}$-band image $\chi_{\mathrm{im}}^{2}(10,276$ degrees of freedom), and a combined estimator defined by

$$
\chi_{\mathrm{comb}}^{2}=\chi_{\mathrm{SED}}^{2}+\chi_{\mathrm{im}}^{2}
$$

The $\chi_{\mathrm{im}}^{2}$ estimators are computed from the sum of the squared pixel-to-pixel differences between the model image and the observed disk image weighted by the estimated noise map, and are normalized by the number degrees of freedom $\nu_{\mathrm{im}}=N_{\mathrm{im}}-N_{\mathrm{par}}$, where $N_{\mathrm{im}}=10286$ is the number of pixels in the image, and $N_{\text {par }}$ the number of model parameters (here 10). The $\chi_{\mathrm{SED}}^{2}$ estimators are computed from the sum of the squared point-by-point differences between the model SED and the observed SED weighted by the data error bars, and are normalized by the number degrees of freedom $\nu_{\mathrm{SED}}=$ $N_{\mathrm{SED}}-N_{\mathrm{par}}$, where $N_{\mathrm{SED}}=19$ is the number of data points in the SED, and $N_{\mathrm{par}}=10$.

Table 4 summarizes the parameters we varied in our modeling, as well as the parameters and chi square values of the models that best fit the SED, the $L^{\prime}$-band image, and the two data sets together. The SED and image of the model that best fit the observed SED and NIRC2 $L^{\prime}$-band image of HD 141569 A simultaneously are also presented in Figure 4. This best model provides an excellent compromise that adequately fits both the measured system SED and the NIRC2 $L^{\prime}$-band image of the inner disk, as demonstrated by its $\chi_{\mathrm{SED}}^{2}$ and $\chi_{\text {im }}^{2}$ very close to their respective best values. The main differences between the best model and our data are the non centro-symmetric features in the $L^{\prime}$-band image discussed in Section 3, which cannot be properly fit by our symmetric disk model: the N-S brightness asymmetry and the arc at the southern extremity of the disk.

We used the Bayesian formalism to determine the validity range of each parameter around their best values (see e.g., Pinte et al. 2008; Milli et al. 2015). Under the assumption that the noise affecting our independent data sets follows a Gaussian distribution, the probability of occurrence of a given model given our data is related to the chi square value by the formula:

$$
P \propto \exp \left(-\frac{\chi^{2}}{2}\right)
$$

By normalizing the probabilities of our models by the sum of the probabilities over the entire grid, we computed the relative probability of each model, given our data and the grid of models. We present in Figure 5 the probability distributions obtained by marginalizing the relative probabilities over each parameter value, for each of the chi-square estimators.

As expected, this analysis shows that the SED alone poorly constrains the geometry of the system, and the $L^{\prime}$-band image alone poorly constrains the dust properties. The probability distribution analysis presented in Figure 5 indicates that the dust mass in the disk is not well constrained by our image (flat probability distribution), but is sharply constrained by the SED. We found a most probable value of $2.0 \mathrm{e}-7 M_{\odot}$, consistent with the value constrained by Thi et al. (2014), and which confirms that the inner disk is much less massive than the outer rings. The grain size ranges from $0.1 \mu \mathrm{m}$ to $10 \mathrm{~mm}$, also mostly constrained by the SED, and consistent with values found in Thi et al. (2014). We note that the most probable value for $a_{\max }$ reaches the highest value allowed in our grid and is thus probably a lower limit. This demonstrates that the disk is composed of large grains, typical of an evolved debris disk. Given the large grain size at the tail of the distribution $\left(a_{\max }=10 \mathrm{~mm}\right)$, most of the mass in the $M_{\text {dust }}$ parameter is contained in the millimeter-size grains, as also noted by Thi et al. (2014). Our $L^{\prime}$-band image mainly helps constraining the geometrical parameters of the disk, with most likely values of 20 au for the scale height $H_{0}$ at a radius of 100 au from the star, of -0.5 for the surface density exponent $\alpha$ (the disks spreads outwards as a consequence of the abundance of small grains, which must have eccentric orbits), 90 au for the outer radius of the disk $R_{\text {out }}$ consistent with thermal infrared data (Fisher et al. 2000; Marsh et al. 2002), and 20 au for the inner disk radius $R_{\mathrm{in}}$, although all chi-square estimators suggest that this is an upper limit, given that we do not detect the inner boundary of the disk in our image. We note that none of our data sets enable us to constrain the flaring coefficient of the disk. Finally, 

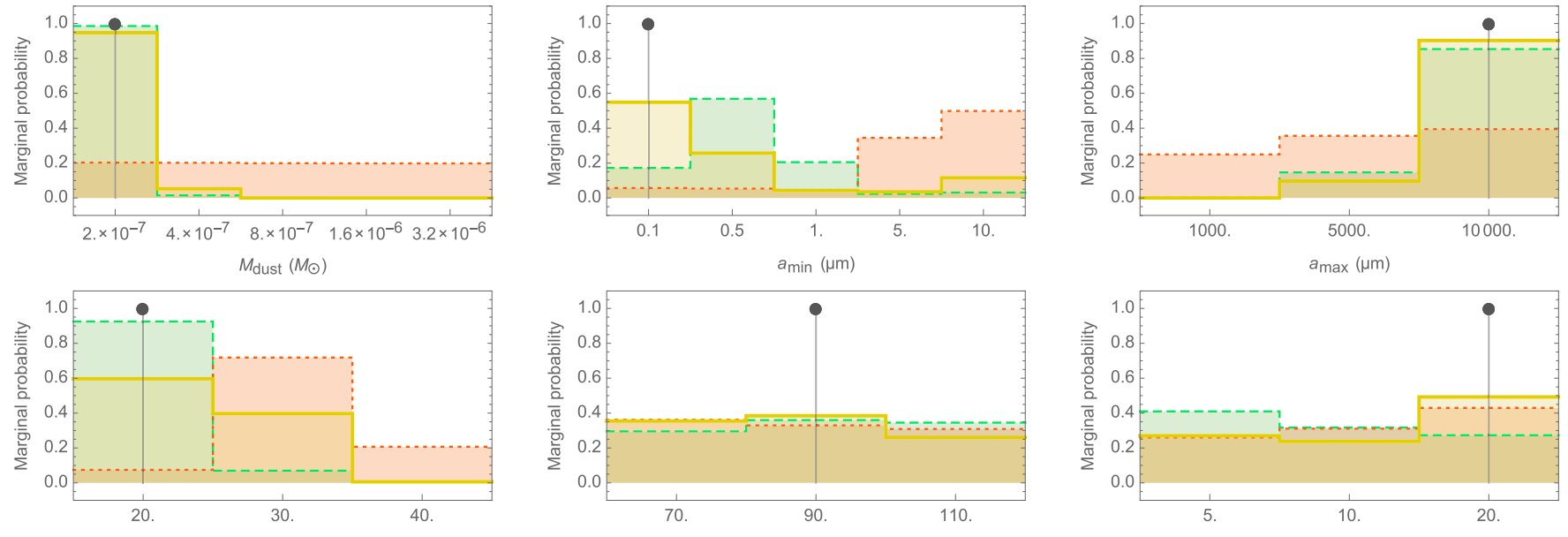

$R_{\text {in }}(\mathrm{AU})$
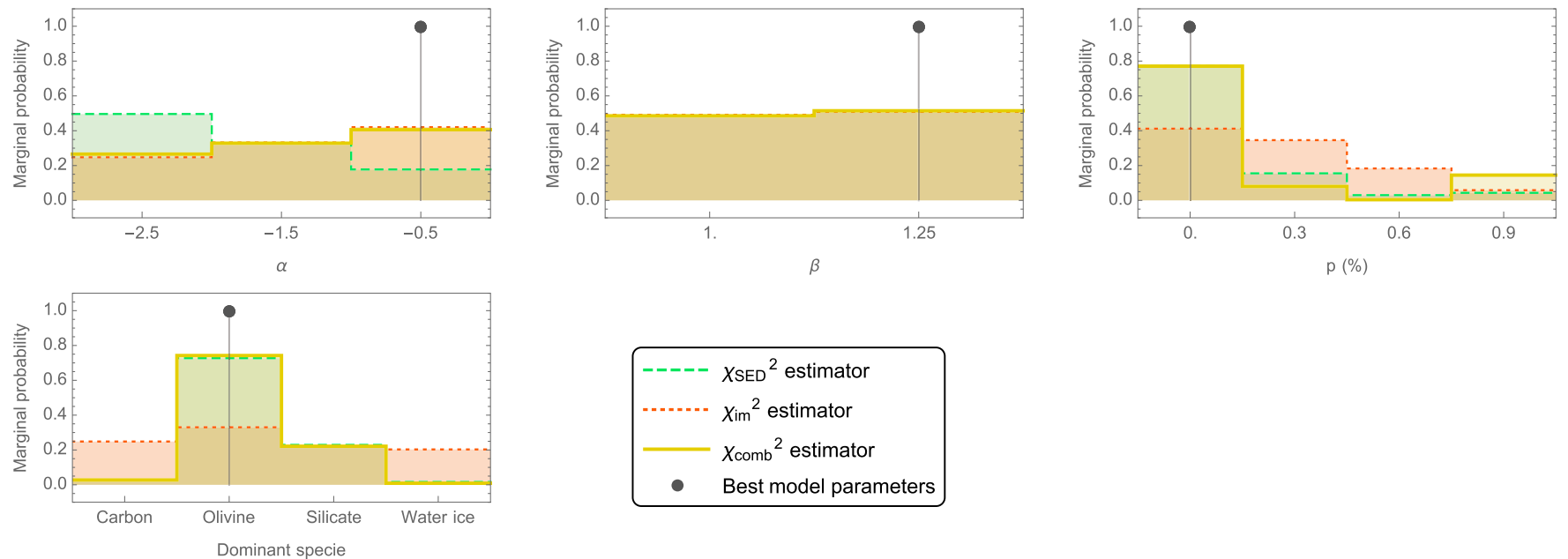

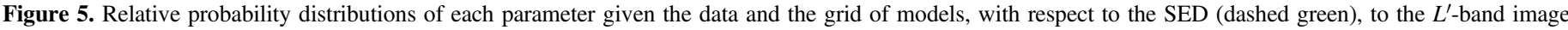
(dotted red), and to both data sets simultaneously (solid yellow). The black dots mark the parameter values of the best model. See text for details.

all three of our goodness-of-fit estimators point with a high probability toward a composition of non-porous olivine grains for the inner disk of the system, as was assumed for the outer rings by Thi et al. (2014).

\section{GIANT PLANET DETECTION LIMITS}

The sensitivity of the Keck/NIRC2 vortex coronagraph to detect giant exoplanet is very good. In Section 3 we reported the detection of a putative and very faint point-like source. If real and associated, the source would hypothetically correspond to a $10 M_{J}$ companion, assuming the BT-SETTL evolutionary model from Allard (2012).

Ignoring the - only-candidate point source present in the data and described in Section 3, we computed our detection limits in and beyond the region of the image covered by the extended disk, using RDI and PCA as described in Section 2. Our reference PSF was constructed using five principal components. Our $5 \sigma$ sensitivity is uniform and close to $\simeq 5$ $M_{J}$ (see Figure 6), assuming the BT-SETTL evolutionary model from Allard (2012). Following Mawet et al. (2014), we accounted for small sample statistics penalties at small inner working angles using the Student t-distribution.

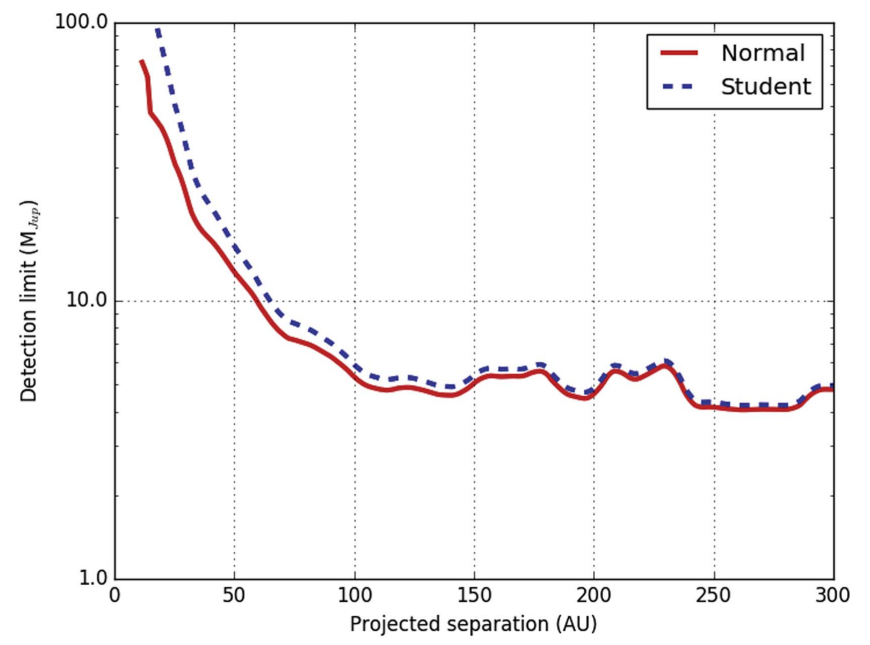

Figure 6. Detection limits $(5 \sigma)$ around HD 141569 A in units of Jupiter mass. The inner working distance is about $10 \mathrm{au}$. The first $100 \mathrm{au}$ are contaminated by the disk scattered light. Far from the star and outside the inner disk, our detection limit is close to $4-5 M_{\text {Jup }}$. We use the BT-SETTL evolutionary model of Allard (2012). The red plain curve shows the traditional $5 \sigma$ detection limits, while the dashed blue curve shows the same detection limits corrected for small sample statistics effect using the Student t-distribution as in Mawet et al. (2014). 


\section{DISCUSSION}

The complex circumstellar environment of five-Myr-old HD $141569 \mathrm{~A}$ is undergoing dramatic changes, evolving from the protoplanetary stage to debris disk. Gas and dust are being processed by physical interactions related to stellar evolution and planetary formation: grain growth, dust settling, gas photoevaporation, Poynting-Robertson drag, and blowout by radiation pressure. This transition is occurring over the timescales of giant planet formation, with its minutiae determining the architecture of the future planetary system. Our detailed analysis sheds new light on the inner disk component around HD 141569 A. Our lower limit on the maximum dust grain size is $10 \mathrm{~mm}$, which is reminiscent of an older debris disk, and shows that significant grain growth has already occurred for this relatively young system. On the other hand, the minimum grain size constrained by our analysis, $0.1 \mu \mathrm{m}$, is well below the blowout size from radiation pressure for HD $141569 \mathrm{~A}(\simeq 4$ $\mu \mathrm{m})$, suggesting a very active disk where collisions are constantly replenishing the reservoir of small grains. The inner disk has some properties of a young protoplanetary disks, and it may not have reached collisional equilibrium yet. Moreover, the presence of small grains being blown out is consistent with our finding of -0.5 for the surface density exponent $\alpha$, which indicates that the disks spread outwards due to the abundance of small grains on eccentric orbits.

Contrary to the conclusions of Li \& Lunine (2003), our best fit to the SED and $L^{\prime}$-band image shows with a high confidence level that the inner disk is composed of a much simpler grain structure and composition. Confirming the assumptions adopted in the disk model used in Thi et al. (2014), we find that non-porous olivine grains have a much higher marginal probability than any of the porous carbonaceous, silicate, and water-ice mixtures considered here. Our data and analysis thus indicates that the inner disk seems indeed devoid of water ice. The fact that we find non-porous grains is also suggestive of asteroidal grains, as opposed to fluffy icy cometary aggregates found, for instance, in older debris disks such as HD 181327 (Lebreton et al. 2012). The composition of the inner disk does not differ significantly from the outer disks, as presented in Thi et al. (2014), suggesting a common origin.

It is interesting to compare our $L^{\prime}$-band data and analysis with the HST/STIS detection presented by Konishi et al. (2016), and tempting to assume that both detections overlap and see the same inner disk component. In reality, there is no significant overlap between our $L^{\prime}$-band data and the optical image, as the disk is not detected beyond 70 au in our $L^{\prime}$-band image, and the HST/STIS detection of Konishi et al. (2016) ranges from 65 to $100 \mathrm{au}$ along the disk major axis. The $L^{\prime}$ band data thus probe much smaller projected separations and a different dust population with larger grains than seen in the STIS visible image. As our MCFOST model also generates optical images, we decided to compare our best-fit model synthetic image to the $H S T /$ STIS data. We found that the predicted flux is brighter than the STIS image. Two reasons can be invoked to explain the discrepancy: the phase function chromatic behavior of our dust particles is not well described by the Mie theory, as discussed in previous studies (Schneider et al. 2006; Hedman \& Stark 2015; Milli et al. 2015), and/or the dust population seen at optical wavelengths is different from the dust population seen in the $L^{\prime}$ band.

The intermediate near-infrared wavelengths probed by the SPHERE data presented in Perrot et al. (2016) offers an interesting perspective. The disk morphology in the SPHERE images shows a broken narrow ring with multiple point sources, none of which are superimposed with our data. These image features are likely the consequence of the high-pass filtering characteristics of ADI, which our RDI reduction prevents (Milli et al. 2012). Nevertheless, the broken ring structure resolved in the SPHERE data sits right at the transition between our $L^{\prime}$-band image and the optical HST/ STIS image. The prominent feature in the near-infrared images seemingly marks a contrasted zone in the surface brightness, reminiscent of a break in dust population characteristics. It is thus likely that the optical image probes a tenuous population of very small grains that are blown out of the inner disk by stellar radiative pressure, as is common at these spatial scales for disks around the same age and evolutionary stage (Milli et al. 2014, 2015). The current picture of the HD 141569 A inner system shows a very active inner component up to $\simeq 70 \mathrm{au}$, where collisions dominate, creating very small grains, and an outward progression in small dust grain location from the $L^{\prime}$ band to the $H$ band (VLT/ SPHERE image) to the visible (HST/STIS image), likely indicative of dust blowout.

Massive companions orbiting exterior to and inclined to a circumstellar disk have recently been shown to excite the disk particle eccentricities and inclinations via the Kozai-Lidov mechanism, producing dust (Nesvold et al. 2016). This mechanism has also been shown by the same author to shepherd rings, providing an alternative scenario to the classical eccentric planet orbiting interior to the disk that stirs the larger bodies in the belt, and produces dust via inter-particle collisions. It is possible that the set of concentric heavily disturbed rings of HD $141569 \mathrm{~A}$ are, at least in part, the results of complex dynamical secular interactions with outer companions. We also note that the spiral-like features seen on multiple scales around HD 141569 A can also be caused by the presence of interior or exterior companions (including the two M-dwarf companions) launching density waves (Dong et al. 2015, 2016). Weinberger et al. (2000) argues that the two other companion stars (types M2 and M4) both around $900 \mathrm{au}$ away in projected separation are associated with HD 141569 A, forming a triple system. However, Reche et al. (2009) demonstrated that the radial velocity measurements of Weinberger et al. (2000) are most likely incorrect, and they used the radial velocity from the $\mathrm{CO}$ line instead, obtaining a more accurate result. The new measurement indicates that the companion pair likely flew by. The flyby scenario, while attractive, still cannot explain the gaps between the two outer rings (Ardila et al. 2005).

\section{CONCLUSION}

We have presented a new $L^{\prime}$-band coronagraphic image of the third disk component around HD 141569 A. The image was obtained during the commissioning of the $L^{\prime}$-band vortex coronagraph installed in Keck/NIRC2. A simple disk model was fitted to the data and yielded a parent body belt location at $r=39$ au. No significant offsets between the disk and the star were observed.

A more complex model generated with the radiative transfer code MCFOST was used to fit both the SED and the new $L^{\prime}$ band image and constrain the dust properties and disk morphology, revealing that the disk is composed of nonporous olivine dust grains, extending from below 20 au and up 
to $90 \mathrm{au}$. We found a total dust mass of $2 \mathrm{e}-7 M_{\odot}$ for grain sizes ranging from $0.1 \mu \mathrm{m}$ to $10 \mathrm{~mm}$. The gas mass is therefore $2 \mathrm{e}-5 M_{\odot}$, consistent with Thi et al. (2014). This population of grains is interior and differs from the population probed in the optical by HST/STIS (Konishi et al. 2016). Interestingly, the ring structure detected in the near-infrared by VLT/SPHERE sits right at the transition between the inner disk component dust population probed by our data and the tenuous small-grain population that is most likely blown out of the inner disk and seen in the HST/STIS image.

Our data and multiwavelength analysis constrained the dust composition of the newly discovered inner disk component of HD 141569 A for the first time. Our analysis reveals that this five-Myr-old disk already agglomerated micron-sized olivine dust grains, similar to evolved debris disks. Studying both the composition of dust grains and their distribution in young transitional disks is key to understanding the last stage of the formation of rocky and giant planets.

Based on observations made at the W.M. Keck Observatory, which is operated as a scientific partnership among the California Institute of Technology, the University of California and the National Aeronautics and Space Administration. The Observatory was made possible by the generous financial support of the W.M. Keck Foundation. We would like to acknowledge J.-C. Augereau for the development and the sharing of the GRaTer disk modeling tool. Support for this work was provided by NASA through Hubble Fellowship grant \#HST-HF2-51355.001-A awarded by the Space Telescope Science Institute, which is operated by the Association of Universities for Research in Astronomy, Inc., for NASA, under contract NAS5-26555.

Facilities: W.M. Keck Observatory, Keck II.

Software: GRaTer (Augereau et al. 1999; Lebreton et al. 2012), MCFOST (Pinte et al. 2006, 2009).

\section{REFERENCES}

Absil, O., Milli, J., Mawet, D., et al. 2013, A\&A, 559, L12

Allard, F., Homeier, D., Freytag, B., \& Sharp, C. M. 2012, in EAS Ser. Vol. 57, Low-Mass Stars and the Transition Stars/Brown Dwarfs, ed. C. Reylé, C. Charbonnel, \& M. Schultheis (Les Ulis: EDP Sciences), 3

Ardila, D. R., Lubow, S. H., Golimowski, D. A., et al. 2005, ApJ, 627, 986

Augereau, J.-C., Lagrange, A.-M., Mouillet, D., \& Ménard, F. 1999, A\&A, 350, L51
Augereau, J. C., Lagrange, A. M., Mouillet, D., Papaloizou, J. C. B., \& Grorod, P. A. 1999, A\&A, 348, 557

Biller, B. A., Liu, M. C., Rice, K., et al. 2015, MNRAS, 450, 4446

Cardelli, J. A., Clayton, G. C., \& Mathis, J. S. 1989, ApJ, 345, 245

Choquet, É., Perrin, M. D., Chen, C. H., et al. 2016, ApJL, 817, L2

Clampin, M., Krist, J. E., Ardila, D. R., et al. 2003, AJ, 126, 385

Currie, T., Grady, C. A., Cloutier, R., et al. 2016, ApJL, 819, L26

Defrère, D., Absil, O., Hinz, P., et al. 2014, Proc. SPIE, 9148, 91483X

Delacroix, C., Absil, O., Forsberg, P., et al. 2013, A\&A, 553, A98

Dohnanyi, J. S. 1969, JGR, 74, 2531

Dong, R., Zhu, Z., Fung, J., et al. 2016, ApJL, 816, L12

Dong, R., Zhu, Z., Rafikov, R. R., \& Stone, J. M. 2015, ApJL, 809, L5

Dorschner, J., Begemann, B., Henning, T., Jaeger, C., \& Mutschke, H. 1995, A\&A, 300, 503

Draine, B. T., \& Lee, H. M. 1984, ApJ, 285, 89

Fisher, R. S., Telesco, C. M., Piña, R. K., Knacke, R. F., \& Wyatt, M. C. 2000, ApJL, 532, L141

Hedman, M. M., \& Stark, C. C. 2015, ApJ, 811, 67

Henyey, L. G., \& Greenstein, J. L. 1941, ApJ, 93, 70

Huby, E., Baudoz, P., Mawet, D., \& Absil, O. 2015, A\&A, 584, A74

Konishi, M., Grady, C. A., Schneider, G., et al. 2016, arXiv:1601.06560

Lebreton, J., Augereau, J. C., Thi, W. F., et al. 2012, A\&A, 539, A17

Li, A., \& Greenberg, J. M. 1998, A\&A, 331, 291

Li, A., \& Lunine, J. I. 2003, ApJ, 594, 987

Marsh, K. A., Silverstone, M. D., Becklin, E. E., et al. 2002, ApJ, 573, 425

Mawet, D., Absil, O., Delacroix, C., et al. 2013, A\&A, 552, L13

Mawet, D., Mennesson, B., Serabyn, E., Stapelfeldt, K., \& Absil, O. 2011, ApJL, 738, L12

Mawet, D., Milli, J., Wahhaj, Z., et al. 2014, ApJ, 792, 97

Mawet, D., Riaud, P., Absil, O., \& Surdej, J. 2005, ApJ, 633, 1191

Mawet, D., Serabyn, E., Stapelfeldt, K., \& Crepp, J. 2009, ApJL, 702, L47

Mazoyer, J., Boccaletti, A., Choquet, E., et al. 2016, ApJ, 818, 150

Milli, J., Lagrange, A. M., Mawet, D., et al. 2014, A\&A, 566, A91

Milli, J., Mawet, D., Pinte, C., et al. 2015, A\&A, 577, A57

Milli, J., Mouillet, D., Lagrange, A. M., et al. 2012, A\&A, 545, A111

Nesvold, E. R., Naoz, S., Vican, L., \& Farr, W. M. 2016, ApJ, 826, 19

O’Donnell, J. E. 1994, ApJ, 422, 158

Perrot, C., Boccaletti, A., Pantin, E., et al. 2016, arXiv:1605.00468v1

Pinte, C., Harries, T. J., Min, M., et al. 2009, A\&A, 498, 967

Pinte, C., Ménard, F., Duchêne, G., \& Bastien, P. 2006, A\&A, 459, 797

Pinte, C., Padgett, D. L., Ménard, F., et al. 2008, A\&A, 489, 633

Pueyo, L. 2016, ApJ, 824, 117

Reche, R., Beust, H., \& Augereau, J. C. 2009, A\&A, 493, 661

Rouleau, F., \& Martin, P. G. 1991, ApJ, 377, 526

Schneider, G., Silverstone, M. D., Hines, D. C., et al. 2006, ApJ, 650, 414

Serabyn, E., Huby, E., Matthews, K., et al. 2016, AJ, 153, 43

Serabyn, E., Mawet, D., \& Burruss, R. 2010, Natur, 464, 1018

Soummer, R., Pueyo, L., \& Larkin, J. 2012, ApJL, 755, L28

Thi, W. F., Pinte, C., Pantin, E., et al. 2014, A\&A, 561, A50

van Leeuwen, F. 2007, A\&A, 474, 653

Vargas Catalan, E., Huby, E., Forsberg, P., et al. 2016, arXiv:1610.05065

Weinberger, A. J., Becklin, E. E., Schneider, G., et al. 1999, ApJL, 525, L53

Weinberger, A. J., Rich, R. M., Becklin, E. E., Zuckerman, B., \& Matthews, K. 2000, ApJ, 544, 937 